\title{
EDITORIAL
}

\section{The role of sibutramine in weight management; Symposium held on 5-6 April 2001, Rome, Italy}

This supplement to the International Journal of Obesity and Related Metabolic Disorders contains manuscripts based on presentations to a symposium, 'The Role of Sibutramine in Weight Management' held on 5-6 April 2001 in Rome, Italy. The symposium, sponsored by Abbott Laboratories, was held to coincide with the European launch of sibutramine, the first of a new generation of weight management drugs indicated for use as adjunctive therapy within a comprehensive obesity management programme.

Obesity is currently a remarkably prevalent disease in Western society and an escalating global healthcare problem. The WHO issued an expert document in 2000, declaring obesity as 'the biggest unrecognised health problem' facing today's society. ${ }^{1}$ The obesity 'pandemic' is also reflected in the signing, by some 51 national ministers of health in the European region of $\mathrm{WHO}$, of an International Nutrition and Diet Action Plan that prioritises obesity as a major, and largely untackled, health threat in this new millennium.

We estimated that more than 81 million are clinically obese in the 20 European countries whose experts contributed to the meeting, that is with a body mass index (BMI) greater than $30 \mathrm{~kg} / \mathrm{m}^{2}$. Subjects are considered overweight with a BMI of between 25 and $30 \mathrm{~kg} / \mathrm{m}^{2}$ and, using this criterion, there are clearly at least an equal number of additional patients for whom being overweight poses considerable health threats. ${ }^{1}$

The symposium proceedings reported here highlight the importance of obesity as a disease in its own right and as a comorbid condition affecting the course and prognosis of common metabolic and cardiovascular diseases such as diabetes, hypertension and dyslipidaemia. The availability of pharmacotherapy to complement diet and exercise regimens offers new opportunities for all those involved in the management of obesity. In plenary sessions and during workshops, speakers and delegates explored the clinical data on sibutramine and shared their experiences of realistic and workable weight management systems, in order to contribute to a blueprint for best clinical practice with sibutramine.

There was widespread agreement that more needs to be done to educate not only the public, but also health professionals who need to recognise obesity as a chronic disease worthy of long-term management. Delegates left the symposium committed to improving education and training in the prevention and management of obesity. They gained an in-depth understanding of sibutramine and what to expect from this novel agent in clinical practice-insights reiterated in detail for the reader of these proceedings.

A series of key recommendations concerning the use of sibutramine as adjunct therapy in weight management programmes was proposed, tackling the many different aspects of the patient management process, from initial recruitment and patient assessment through to initiation and assessment of treatment and management programmes. This blueprint for clinical practice has been devised with the primary care physician very much in mind, and offers clear, stepwise guidance and support for all healthcare professionals who wish to consider sibutramine as part of their weight management programme for the control of obesity.

\section{References}

1 WHO. Obesity: preventing and managing the global epidemic. Report of a WHO Consultation. WHO Technical Report Series, no. 894. WHO: Geneva, 2000

WPT James

Director, Public Health Policy Group, Chairman, International Obesity Task Force, London, UK 